\title{
Myoadenylate deaminase deficiency associated to a liver cirrhosis: A possible cause of neurological dysfunction resembling a hepatic encephalopathy
}

\author{
Marco Fiore ${ }^{1}$, Lorenzo Andreana ${ }^{2}$, Nicola Coppola ${ }^{3}$, Annarosa Floreani $^{4}$ \\ 1. Infectious Disease Unit, University Hospital of Trieste, Italy Piazzale Ospitale, Trieste, Italy. 2. Department of \\ Emergency Medicine and First Aid, Ospedale di Palmanova, Palmanova, Italy. 3. Department of Mental Health and Public \\ Medicine, Infectious Diseases Section, Second University of Naples - Via L. Armanni, Naples, Italy. 4. Department of \\ Surgery, Oncology and Gastroenterology, University of Padova, Via Giustiniani, Padua, Italy. \\ Correspondence: Annarosa Floreani. Address: 4Department of Surgery, Oncology and Gastroenterology, University of \\ Padova, Via Giustiniani, 2 - 35128, Padua, Italy. E-mail: annarosa.floreani@unipd.it \\ Received: January 5, 2015 \\ DOI : $10.5430 /$ crcp.v2n2p95 \\ Online Published: February 13, 2015 \\ Accepted: February 11, 2015 \\ URL: http://dx.doi.org/10.5430/crcp.v2n2p95
}

\section{Abstract}

We report a clinical case of a 44-year-old Caucasian male presenting with decompensated HCV-related liver cirrhosis and clinical symptoms involving the central nervous system, particularly muscular spasms and loss of consciousness. Hepatic encephalopathy (HE) was ruled out on the basis of normal levels of ammonia and normal psychometric and neurophysiological tools. A muscle biopsy revealed alterations consistent with homozygosity for myoadenylate deaminase deficiency (MADD). We hypothesize that the liver failure is responsible for an excess of adenosine monophosphate (AMP) which may exert an up-regulation in the expression of glutamine synthetase (GS) in astrocytes, with the consequence of astrocyte swelling, which is one of the steps in the onset of cerebral edema.

\section{Key words}

Myoadenylate deaminase deficiency, Cirrhosis, Cryoglobulinemia

\section{I ntroduction}

Hepatic encephalopathy (HE) is a frequent complication and one of the most debilitating manifestations of liver disease, severely affecting the lives of patients and their caregivers ${ }^{[1]}$.

It is characterized by a wide spectrum of nonspecific neurological and psychiatric abnormalities. The diagnosis may be difficult, especially in case of normal ammonia levels. In fact, finding normal ammonia levels in a confused, disorientated or comatose cirrhotic patient should prompt immediate search for alternative causes of neuropsychiatric dysfunction ${ }^{[2]}$. The differential diagnosis includes several conditions including overt hepatic encephalopathy or acute confusional status due to diabetes, alcohol, drugs, neuro-infections, electrolyte disorders, non-convulsive epilepsy, psychiatric disorders, intracranial bleeding and stroke, dementia, brain lesions, and obstructive sleep apnea ${ }^{[1]}$. 
Myoadenylate deaminase deficiency (MADD) is a recessive genetic metabolic disorder of skeletal muscle affecting approximately $2 \%$ of the caucasian population ${ }^{[3,4]}$. In the homozygous form, MADD is characterized by the absence of any biochemical and histochemical adenosine monophosphate (AMP) deaminase activity ${ }^{[5]}$; there are different clinical pictures ranging from asymptomatic to symptomatic patients with exercise-induced myalgia. It is unclear, however, whether these individuals are at risk of developing complications. Moreover, no data are available on the association between MADD and liver cirrhosis. We describe the neurologic symptoms resembling hepatic encephalopathy in a patient with liver cirrhosis and homozygous for the AMPD gene mutation.

\section{Case report}

A 44-year-old Caucasian man was admitted in April 2007 to hospital for his first episode of slight ascites and lower-limb edema. In his past history he underwent a maxillary surgery for removing a sinus cyst in 2002 . At that time he was diagnosed as having a chronic hepatitis $\mathrm{C}$ virus $(\mathrm{HCV})$, genotype $3 \mathrm{a}$ with a viral load detectable at real-time PCR of $1.8 \times$ $105 \mathrm{IU} / \mathrm{ml}$. He had no co-infection with HIV, and was positive for anti-hepatitis B surface and anti-hepatitis B core antibodies. He had no history of drug use, and was a moderate drinker (1-2 units per day until 2002).

In June 2005 he underwent liver biopsy, which revealed a liver cirrhosis (grade 10, stage 6, according to Ishak's scoring system), and treatment with standard pegylated interferon alpha-2a (180 $\mu \mathrm{g} / \mathrm{week})$ plus ribavirin $(1,200 \mathrm{mg} /$ day $)$ was started thereafter. Antiviral therapy was discontinued after 45 days, due to marked anemia (hemoglobin $\sim 6 \mathrm{~g} / \mathrm{dl}$ ) and fatigue. Red blood count normalized after discontinuation of antiviral treatment, but fatigue worsened and the patient developed nausea, anorexia, weight loss, and peripheral muscle spasms with a cranial-caudal progression. Neurological symptoms (irritability, diffuse muscle spasms and loss of consciousness) developed; the patient gradually regained consciousness within a few minutes, but the muscle pain persisted for 4-5 hours. Neurological examination showed proximal and distal weakness in all limbs with no sensory loss. Deep tendon reflexes were normal, with a slight reduction in the right Achilles tendon reflex.

Hemolysis and acute intermittent porphyria were ruled out on the basis of specific blood and urine tests. Magnetic nuclear resonance of the brain, CT scan of the brain, electroencephalogram, and nerve conduction studies (EMG) suggested a diagnosis of multifocal motor neuropathy. Abdomen ultrasound revealed ascites and signs of liver cirrhosis with no evidence of hepatocellular carcinoma.

Laboratory tests showed: alanine aminotransferase, $62 \mathrm{U} / \mathrm{L}$ (upper normal limit [unl] $35 \mathrm{U} / \mathrm{L}$ ); aspartate aminotransferase $125 \mathrm{U} / \mathrm{L}$ (unl 40); total bilirubin $6.54 \mathrm{mg} / \mathrm{dl}$ (normal range 0.2-1.2); alkaline phosphatase $239 \mathrm{U} / \mathrm{L}$ (unl 128); albumin

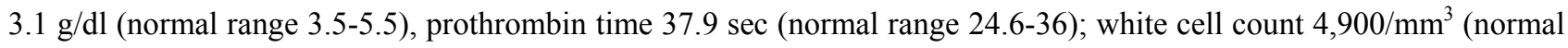
range 4,800-10,800); hemoglobin 12.2g/dL (normal range 13.5-18); and platelet count $125,000 / \mathrm{mm}^{3}$ (normal range 130,000 to 400,000 ); ammonia $136 \mu \mathrm{g} / \mathrm{dl}$ (normal range 25-94), creatine phosphokinase (CK) $96 \mathrm{U} / \mathrm{L}$ (normal range $10-80$ ); cryocrit value $>5 \%$. Further ammonia tests were always found within the normal range.

A skin cryoglobulinemic lesions were present peripherally: a palpable purpura and skin ulcers. A muscle biopsy showed alterations consistent with MADD homozygosis, with absence of either perivascular inflammatory infiltrate and perifascicular muscle fiber atrophy. A nerve biopsy showed a cryoglobulinemic neuropathy characterized by focal perineurial thickening and inflammation with endoneurial microvessels exhibiting endothelial cell swelling, laminal narrowing, and basal lamina reduplication.

\section{Discussion}

The interest in the present case report is the differential diagnosis of a hepatic encephalopathy in a patient with decompensated cirrhosis. The described co-existence of MDD and liver cirrhosis is rather due to chance, as there is no 
common pathogenic mechanism linking the two conditions. In our patient the neurologic syndrome was not explained by a typical encephalopathy, but was correlated with the association between liver disease and the MADD. Indeed, MADD is considered a common cause of exercise-induced myopathy and is probably the most common cause of metabolic myopathy in humans. Fishbein et al. ${ }^{[6]}$ found $1 \%-2 \%$ of all muscle biopsies submitted for pathological examination deficient in AMPD activity. Although the clinical relevance of MADD has recently been questioned, MADD is a clinically heterogeneous metabolic disorder with a clinical spectrum that can range from no symptoms to mild exercise-induced myalgia. MADD is also found in association with other distinct neuromuscular disorders, in which case it may be triggered partly by pathological changes or act in synergy with another metabolic disease ${ }^{[7]}$.

Biochemically, MADD coincides with an excess of AMP accumulating in the cell and being carried by the blood to the liver to be metabolized, or to the kidneys to be excreted. Consequently, the deficiency causes two different effects: first of all, significant amounts of AMP remain in the blood; secondly, ammonia may be not released when the glutamine synthetase (GS) is up-regulated by an excess of AMP. It is should be hypothesized that in our patient, an excess of AMP was transported via blood to the liver, where an impairment of liver function was present, due to cirrhosis. Thus, the anomalous neurologic syndrome, resembling a HE, but with blood ammonia concentration only slightly abnormal, and without the characteristic changes in psychometric tests and in EEG, can be correlated with the excess of AMP.

In HE ammonia enters the brain via diffusion from the blood or cerebrospinal fluid, and its removal relies almost entirely on the GS localized mainly in the astrocytes ${ }^{[8]}$. The metabolism of ammonia to glutamine gives the glutamine an osmotic effect and this leads to an osmotic disruption with astrocyte swelling and brain edema ${ }^{[9]}$. Since patients with cirrhosis show signs of low-grade cerebral edema ${ }^{[10]}$, we hypothesize that astrocyte swelling is due to the pathogenic action of ammonia in HE syndrome ${ }^{[1]]}$. Over the past 20 years, experimental studies have shown that GS expression is upregulated by AMP in primary cultures of astrocytes from the brains of neonatal rats ${ }^{[12]}$. Although no corresponding data are available for humans, we suggest that GS up-regulation can prompt an atypical neurological presentation of the HE syndrome in individuals with MADD and a reduced hepatic functional reserve, as in our patient.

Another interesting aspect of the present case was the concomitant cryoglobulinemic neuropathy which was diagnosed by the nerve biopsy. HCV chronically infects lymphocytes, specially B cells, triggering non neoplastic, oligoclonal B-cell expansion, yielding cryoglobulins ${ }^{[13]}$. Cryoglobulinemic neuropathy is due to vasa nervorum inflammatory destruction, which may feasibly have induced a muscle stress that led to the clinical expression of MADD with an excess production of AMP $^{[14]}$.

In conclusion, precipitating factors are crucially important in the diagnosis and management of HE.

Although no corresponding data are available for humans, we suggest that GS up-regulation may trigger HE in individuals with chronic liver disease associated with MADD. We hypothesize that MADD should be considered as a possible condition associated in cirrhotic patients with normal levels of ammonia.

\section{References}

[1] Vilstrup H, Amodio P, Bajaj J, et al. Hepatic encephalopathy in chronic liver disease: 2014 Practice Guideline by the American Association for the Study of Liver Diseases and the European Association for the Study of the Liver. Hepatology. 2014; 60: 715-35. PMid: 25042402. http://dx.doi.org/10.1002/hep.27210

[2] Romero-Gomez M, Montagnese S, Jalan R. Hepatic encephalopathy in patients with acute decompensation of cirrhosis and acute-on-chronic liver failure. J Hepatol. 2014 in press.

[3] Gross M. Clinical heterogeneity and molecular mechanisms in inborn muscle AMP deaminase deficiency. J Inherit Metab Dis. 1997; 20: 186-92. PMid: 9211191. http://dx.doi.org/10.1023/A:1005352605421

[4] Fischer S, Drenckhahn C, Wolf C, et al. Clinical significance and neuropathology of primary MADD in C34-T and G468-T mutations of the AMPD1 gene. Clin Neuropathol. 2005; 24: 77-85. PMid: 15803807. 
[5] Sabina RL. Myoadenylate deaminase deficiency. A common inherited defect with heterogeneous clinical presentation. Neurol Clin. 2000; 18: 185-94. http://dx.doi.org/10.1016/S0733-8619(05)70184-5

[6] Fishbein WN, Armbrustmacher VW, Griffin JL. Myoadenylate deaminase deficiency: a new disease of muscle. Science. 1978; 200: 545-8. PMid: 644316. http://dx.doi.org/10.1126/science.644316

[7] Jinnah HA, Sabina RI, Van Den Berghe G. Metabolic disorders of purine metabolism affecting the nervous system. In: Handbook of Clinical Neurology. Edited by O. Dulac, M Lassonde, HB Sarnat. Elsevier; 2013. vol. 13, pages 1827-36.

[8] Norenberg MD, Martinez-Hernandez A. Fine structural localization of glutamine synthetase in astrocytes of rat brain. Brain Res. 1979; 161: 303-10. http://dx.doi.org/10.1016/0006-8993(79)90071-4

[9] Albrecht J, Dolinska M. Glutamine as a pathogenic factor in hepatic encephalopathy. J Neurosci Res. 2001; 65 : 1-5. http://dx.doi.org/10.1002/jnr.1121

[10] Haussinger D, Laubenberger J, vom Dahl S, et al. Proton magnetic resonance spectroscopy studies on human brain myo-inositol in hypo-osmolarity and hepatic encephalopathy. Gastroenterology. 1994; 107: 1475-80. http://dx.doi.org/10.1016/0016-5085(94)90552-5

[11] Haussinger D. Low-grade cerebral edema and the pathogenesis of hepatic encephalopathy in cirrhosis. Hepatology. 2006; 43: 1187-90. PMid: 16729329. http://dx.doi.org/10.1002/hep.21235

[12] Max SR, Landry ME, Zielke HR. Induction of glutamine synthetase by 8-bromo cyclic AMP in primary cultures of rat brain astrocytes. Neurochem Res. 1990; 15: 583-6. PMid: 1977090. http://dx.doi.org/10.1007/BF00973746

[13] Ramos-Casals M, Stone JH, Cid MC, et al. The cryoglobulinaem,ias. Lancet. 2012; 379: 345-360. http://dx.doi.org/10.1016/S0140-6736(11)60242-0

[14] Collins MP. The vasculitic neuropathies: an update. Curr Opin Neurol. 2012; 25: 573-585. PMid: 22918487. http://dx.doi.org/10.1097/WCO.0b013e3283580432 